\title{
The Study on the Application of EPON Technology in Optical Fiber to the Home Broadband Access Network Design
}

\author{
Hechuan $^{1, a}$ \\ ${ }^{1}$ ChongQing College of Electronic Engineering, ChongQing, 401331,China \\ ahechuan023@sina.com
}

Keywords: EPON technology; Optical fiber to the home; Broadband access network; Design

\begin{abstract}
At present, the individual users use ADSL dial-up Internet access methods, but due to the copper price too high, broadband couldn't be more improved, the copper is bound to be abandoned by the society. Some new network providers and operators provide active optical fiber to the community. Due to the new network provider not PSTN network, so the copper coverage area is not be considered. The active optical cable access network is mainly to provide optical fiber to the village, and then combined with the community LAN, ultimately to provide broadband access. This article mainly discusses the EPON technology briefly, and analyzes the optical fiber to the home network model, and the optical fiber to the home broadband access network based on EPON technology has carried on the detailed discussion of the design and implementation and it is able to provide users with high quality, high speed communications services.
\end{abstract}

\section{Introduction}

The current environment, different types of Internet application in rapid development, the application of communications bandwidth demand also increases gradually, combined with national policy of "triple play", make communication broadband network is facing new challenges. Internet access technology in China from the PSTN based on copper twisted pair narrowband dial to the ADSL technology, to the optical fiber to the home access technology, FTTH access in constant transformation, the corresponding access technology has been perfect. Ethernet passive optical network (PON) technology is an important technology in the process of optical fiber to the home, it has high bandwidth, can provide reliable data for the end user, voice, video transmission capacity, together with its mature technology, the cost of falling, promote the general application of optical fiber access. In order to adapt to the trend of the development of the mass, FTTH domestic three big operators have already begun in each area of FTTH, although at present the technology in some aspects and some conditionality, but in the long run, FTTH will obtain the large-scale development in our country. Based on optical fiber to the home business development involves in the process of technology, market, maintenance and other aspects are studied, and puts forward different village, respectively different implementation plan, this paper expounds the advantages of EPON as ideal FTTH access means.

\section{The summary of EPON technology}

EPON technology. Optical fiber access is to use fiber for transmission between the user and bureau of end, and the optical fiber is the only transmission media. Optical fiber access network, including active optical network (AON), passive optical network (PON), passive optical network including optical line terminal (OLT) and optical network unit (ONU), and optical distribution network (ODN), PON technology mainly includes the BPON, EPON and GPON. EPON (Ethernet passive optical network) technology is based on the IEEE802.3 Ethernet technology, is the main variable length data transmission, TDD can provide has symmetrical line transmission rate of up to $1 \mathrm{Gbit} / \mathrm{s}$, and easy to upgrade, EPON system is relatively simple in structure, low cost, has a loose standards, on the other hand, EPON Ethernet is compatible with the existing, therefore do not need additional protocol conversion, in the case of guaranteed QoS, can easily realize communication, 
therefore, can achieve the transparent transmission of user data. The characteristics of EPON technology makes it widely used.

EPON optical fiber to the home network model. Optical fiber to the home through the optical fiber, refers to the only FTTH transmission medium to the network connection to the homes of users, to provide users with video, language, data, information and other business, and can provide users with different demand of broadband communications. Optical fiber itself can provide a higher bandwidth, and has high transmission speed, therefore, optical fiber to the home to be able to provide users with high-quality transport services.

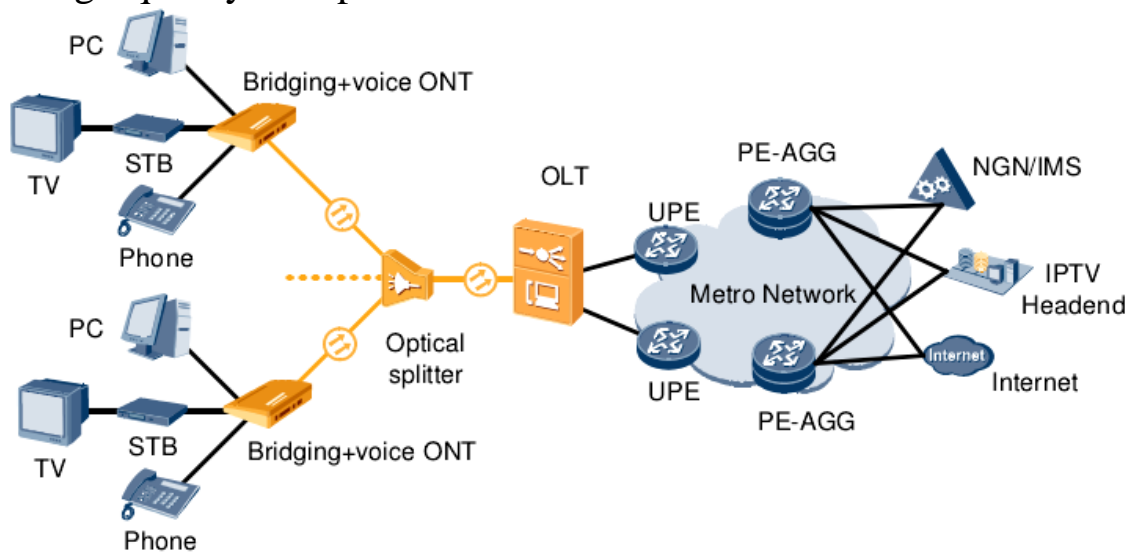

Fig. 1: The bridge type + voice ONT access business networking

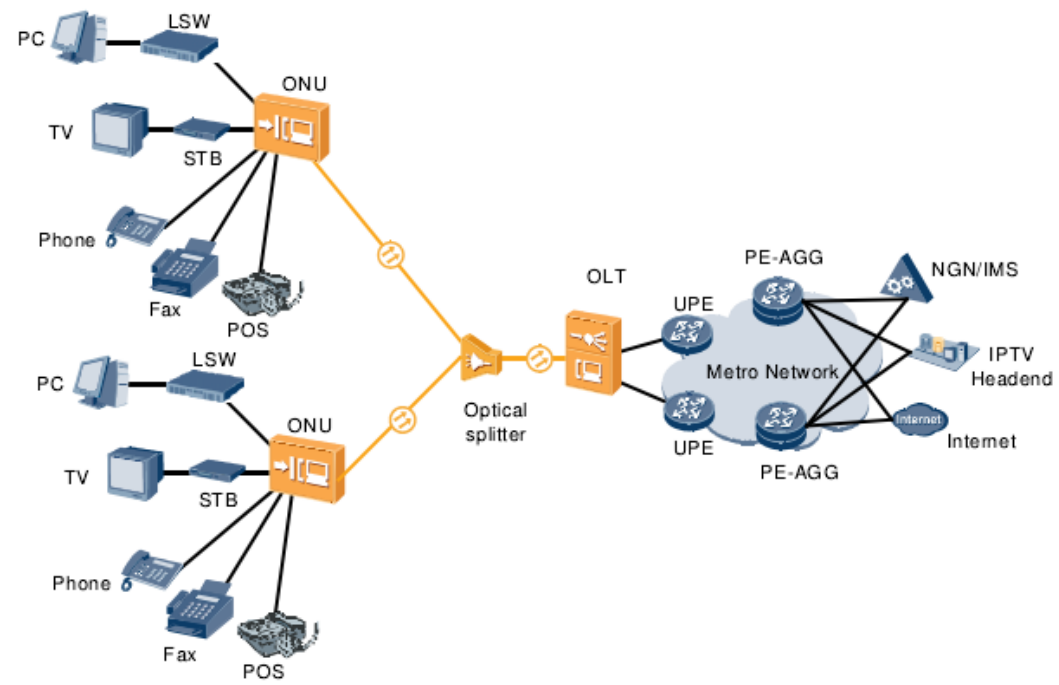

Fig. 2: The gateway ONU networking

\section{Analysis of the traditional access methods}

The copper based community broadband access. At the early stage, provide data on the PSTN business once through the process of the narrowband and broadband. To provide users with narrowband access to the Internet, need a computer to install this way narrow-band modem terminal, but only to provide users with $56 \mathrm{~KB} / \mathrm{s}$ access bandwidth. Although later as technology advances, on PSTN provides the ISDN line of business, improving access bandwidth by times, but not in the true sense of broadband access. The emergence of xDSL technology, however, makes the efficiency of copper to the limit. ADSL technology to provide users with multiple levels of access bandwidth, HDSL technology provides access, VDSL can provide users with higher user requirements, but there is a limiting factor, the length of the copper to within 800 meters.

It is given priority to with active optical cable broadband access. Although the emergence of xDSL technology, makes the copper efficiency to the limit, but with the emergence of ultra-high applications such as video, apparently xDSL technology can't satisfy people growing demand for 
bandwidth. Copper price is more expensive than cable, at the same time, provide the narrow bandwidth than cable, so new operators preferred or fiber-optic access way. This approach can provide users with stable and high bandwidth at the same time, online can't break a higher access bandwidth. If some new compounds with the method of optical fiber to the building, the building corridor switch installation, to provide users with a LAN access. This way, in theory, can provide users with $10 \mathrm{~m}$ exclusive or $100 \mathrm{~m}$ share bandwidth.

The passive network access bandwidth. Traditional active optical fiber to the building adopts way from access to the computer room cloth cable, how many buildings is a village, then how much from the computer access to the building of the fiber core, using passive optical network, only need to in the optical fiber box installed a passive way. In terms of bandwidth, the traditional way of active optical fiber access to the floor if $100 \mathrm{~m}$ to the building, the passive optical network way is $1000 \mathrm{~m}$ to the building, that is to say, can provide users with a wider bandwidth. At the same time also don't have to worry about building equipment of power supply and.

\section{The EPON FTTH technology link detection and signal transmission characteristics}

EPON FTTH technology link detection particularity. Using PON (passive optical network) the topology of the realization of the Ethernet access is a basic feature, EPON FTTH technology in the end of the access network to achieve the user a wide range of coverage. Light shunt coupled device is the hub of the link, it is a signal of power distribution device, into the fiber signal power difference between single out fine signal power $1010 \mathrm{NN}$ ( $\mathrm{N}$ for shunt ratio), if 1:32 divider, single out the fiber power will be smaller than into fiber power about $15 \mathrm{db}$, this to the general line detection using OTRD dynamic range is not enough.

In addition, due to the complexity of by the user access network cabling environment, especially, FTTH fiber from OLT to ONU, the intermediate links are OON, user access point, and the wiring inside the building around the corner, ODN, distance to the user terminal, short, long distance is a few hundred meters, short only a few meters, for the general line detection with 0 TRD the detection precision of the positioning line fault point is not enough. In view of the above wiring environmental complexity and particularity of link segment, need some special instrument to meet the needs of the fault detection.

The EPON technology of signal transmission FTTH needs special testing equipment. In order to realize the three nets as own duty, FTTH is basically in a single fiber transmission three wavelength signal, respectively is $1490 \mathrm{~nm}$, downstream data signals downside TV signal is 1550 $\mathrm{nm}$, uplink signal is $1310 \mathrm{~nm}$. Due to the function of each band to achieve different, during construction and testing of the need for different wavelength signal detection means, the current general power meter lack power test of 1490 - nm wavelength. In addition because of PON network topology and ONU trigger type works, most of the time in ONU not up signal, so the general test $1310 \mathrm{~nm}$ wavelength of power meter cannot used for testing the FTTH1310nm wavelength of EPON technology. The ordinary cable skin line with single core cable on the optical fiber classifier with the method is the hot melt docking. Single-core leather line of fiber-optic access every backplane melt in the FTTH fiber plate of a heat pipe with $0.5 \mathrm{~m}$ end to end, can also choose to mechanical straight plugs and skin line cable directly into the side.

The side FTTH tests method of EPON technology. FTTH system itself has a general fault alarm function, for a certain ONU has no job, will be showed in the system administrator, you can preliminary judgment is the failure of hard or soft. There is rarely FTTH construction supporting line fault location self-checking system, the main reason is that the terminal line is too short, PON point to multipoint topology structure increased the difficulty of the self-checking system of the line, it will also greatly increase the cost of construction. Necessary to complete detection means, of course, is to ensure that the key to the success of a project. In the early stage of the construction, it is necessary to establish the complete each part of the testing data files, such as divider, connector and cable of the original test data. In the process of construction, to link connectivity, link length, two-way end-to-end attenuation (dual wavelength or three wavelength) and bidirectional optical return loss test, and use the visual fault locator initially determined VFL link fiber fracture and 
macro bending failure may occur, and using the optimized PON OTDR compare accurate positioning. If there is a line on-line self-checking system construction, can use $1650 \mathrm{~nm}$ wavelength of light detection, in case of transmission wavelength signal interference.

Which link the total attenuation should satisfy the standard of the design of the corresponding categories, for CATV transmission line construction should prevent reflection for CATV system; the influence of the joints and routes of the return loss is the very high request. Business opened, should control the corresponding indicators for each test, if a business indicators can not meet the requirements, may be associated with the power shortage of related signal, the paragraphs of this request to the appropriate link to verify the basic data such as light power and return loss. Engineering maintenance and troubleshooting, mainly involves the PON optimize the use of OTDR and multi-function power meter. With multi-function power meter for first line fault and preliminary judgment, the equipment failure and can use the visual fault locator VFL identify and macro curved line faults, in the final optimized by PON OTDR business on-line detection wavelength of $1650 \mathrm{~nm}$, accurate locating the fault position.

\section{Summary}

Optical fiber to the home (FTTH) is at present, the development of the network communication goal, but because of the technology, requirements, policies and other factors, has not the large-scale construction and promotion. With the increase of the average user demand for broadband, optical fiber to further reduce the cost, the operators, the competition between the FTTH is becoming a hot spot of attention and focus, attracted the attention of many researchers. Optical fiber communication capacity big, the secrecy good performance and strong anti-jamming capability, make it become the mainstream of modern communication network.

\section{References}

[1] A Broadband Optical Access System with Increased Service Capability Using Dynamic Bandwidth Assignment. ITU-T Recommendation G.983.4 . 2012.

[2] ITU-T. Gigabit-Capable Passive Optical Networks (GPON) :Physical Media Dependent (PMD) Layer Specification. ITU-T Recommendation G 984.2. 2013.

[3] Gigabit-capable Passive Optical Networks (GPON): Transmission Convergence Layer Specification. ITU-T Recommendation,G.984.3. 2013.

[4] Aswir Premadi,Mohammad Syuhaimi Ab-Rahman, Ng Boon Chuan,etal. Protection Scheme ofFiber to the Home Passive Optical Network using Access Control System. Innovative Technologies in Intelligent Systems and Industrial Applications. 2012.

[5] Almeida P,Silva H.Multiservices and multiband optical signal generation for hybrid access networks. Optical Network Design and Modeling. 2011. 